\title{
Ocorrência de Disfunção Temporomandibular em portadores de Doença Pulmonar Obstrutiva Crônica
}

\author{
Eulália S S Pinheiro \\ EBMSP - Escola Bahiana de Medicina e Saúde Pública \& \\ GDSNME - Grupo de Pesquisa em Dinâmica do Sistema \\ Neuromusculoesquelético \\ EBMSP - Escola Bahiana de Medicina e Saúde Pública \& \\ Roberta Gonçalves Gonçalves \\ GDSNME - Grupo de Pesquisa em Dinâmica do Sistema \\ Neuromusculoesquelético \\ Abrahão Fontes Baptista \\ GDSNME - Grupo de Pesquisa em Dinâmica do Sistema \\ Neuromusculoesquelético \& UFBA - Universidade Federal \\ da Bahia \\ Selena Márcia Dubois Mendes \\ EBMSP - Escola Bahiana de Medicina e Saúde Pública \& \\ GDSNME - Grupo de Pesquisa em Dinâmica do Sistema \\ Neuromusculoesquelético \\ Guilhardo Fontes Ribeiro \\ HSI - Hospital de Ensino Santa Izabel \\ EBMSP - Escola Bahiana de Medicina e Saúde Pública \& \\ Kátia Nunes Sá GDSNME - Grupo de Pesquisa em Dinâmica do Sistema \\ Neuromusculoesquelético \\ Feita pelo Corpo Editorial da Bahiana \\ Aprovado em Dez/10.
}

Análise do Manuscrito

\section{Resumo}

Introdução: Estudos sugerem uma possível relação entre a incidência de patologias respiratórias e a ocorrência de Disfunção Temporomandibular (DTM), referindo à obstrução como motivo de alterações na postura craniocervical e mandibular para facilitar a respiração e vencer a maior resistência das vias aéreas. Objetivo: Verificar a ocorrência de DTM em pacientes portadores de Doença Pulmonar Obstrutiva Crônica (DPOC). Metodologia: Estudo observacional de corte transversal realizado em 30 indivíduos de ambos os sexos, com idade entre 40 e 65 anos, portadores de DPOC. A Coleta de Dados consistiu na aplicação dos questionários sociodemográfrico e Anamnésico, para avaliação e graduação da DTM e na avaliação da mobilidade torácica, através da cirtometria. Resultados-Conclusão: Há uma alta prevalência de DTM em portadores de DPOC (90\%) e o grau moderado desta disfunção foi o de maior prevalência. 0 grau de sinais e sintomas de DTM está associado com a gravidade da DPOC.

Palavras-chave: Fisioterapia, Postura, DTM, DPOC

\section{Temporomandibular disorders occurrence in Obstrutive Chronic Disease Patients}

\begin{abstract}
Introduction: Studies suggest a possible relation between the incidence of respiratory pathologies and occurrence of Temporomandibular Disorder (TMD), relating the obstruction as reason of alterations in the craniocervical and mandibular position to facilate the breath and beat the bigger resistance of aerial ways. Objective: To verify the occurrence of the TMD in patients who suffer of Chronic Obstructive Pulmonary Disease (COPD). Methodology: Obsevacional study of tranversal cut to carried in 30 individuals of both the sex, age between 40 and 65 years, carriers of COPD. The collect of the data consisted of the application of a demographic, social and of Anamnesis' Questionnaires to evaluate and graduate the TMD and
\end{abstract}


in the evaluation of the thoracic mobility, through the cirtometria. Results-Conclusion: There is a high prevalence of TMD in patients with COPD $(90 \%)$ and a moderate degree of this disfunction was the bigger prevalence. The degree of signals and symptoms of TMD is associated with the severity of the DPOC.

Key-words: Physiotherapy, Posture, TMD, COPD

\section{La aparición de la disfunción temporomandibular en pacientes con Enfermedad Pulmonar Obstructiva Crónica}

\section{Resumen}

Introducción: Los estudios sugieren una posible relación entre la incidencia de enfermedades respiratorias y la aparición de trastornos temporomandibulares (TTM), en referencia a la obstrucción como causa de los cambios de postura y mandibular craneocervical para facilitar la respiración y para superar la mayor resistencia de las vías respiratorias. Objetivo: Investigar la incidencia de la DM en pacientes con Enfermedad Pulmonar Obstructiva Crónica (EPOC). Metodología: Estudio observacional transversal realizado en 30 individuos de ambos sexos, con edades comprendidas entre 40 y 65 años con EPOC. La recolección de datos fue la administración de los cuestionarios y sociodemográfrico anamnesis para la evaluación y clasificación de la DM y la evaluación de la movilidad torácica a través de cirtometry. Resultados-Conclusiones: Existe una alta prevalencia de la DM en pacientes con EPOC (90\%) y moderado grado de disfunción fue el más prevalente. El grado de signos y síntomas de TTM se asocia con la gravedad de la EPOC.

Palabras-clave: fisioterapia, la postura, DTM, la EPOC

\section{Présence d'un dysfonctionnement temporo chez les patients atteints de bronchopneumopathie chronique obstructive}

\section{Résumé}

Introduction: Des études suggèrent une relation possible entre l'incidence des maladies respiratoires et la survenue de troubles temporo-mandibulaire (ATM), se référant à l'obstruction comme une cause de changements de posture et de la mandibule cranio pour faciliter la respiration et de surmonter la plus grande résistance des voies respiratoires. Objectif: enquêter sur l'apparition de TMD chez les patients avec maladie pulmonaire obstructive chronique (MPOC). Méthodologie: Une étude transversale observationnelle menée dans 30 individus des deux sexes, âgés entre 40 et 65 atteints de MPOC. La collecte des données était d'administrer les questionnaires et l'anamnèse sociodemográfrico pour l'évaluation et le classement des TMD et l'évaluation de la mobilité thoracique par cirtometry. Résultats-Conclusion: Il existe une prévalence élevée de TMD chez les patients atteints de BPCO (90\%) et le degré de dysfonction modérée était la plus répandue. Le degré de signes et symptômes de DTM est associée à la gravité de la BPCO.

Mots-clés: physiothérapie, la posture, DTM, la BPCO

\section{Introdução}

O termo disfunção temporomandibular (DTM) é comumente utilizado para designar alguma doença que acomete músculos mastigatórios, articulação têmporo-mandibular (ATM) ou estruturas adjacentes.1 A etiologia das DTM's é ainda bastante controversa e discutida entre os profissionais da área da saúde (dentistas, médicos e fisioterapeutas), mas é consenso que esta envolve múltiplos fatores. 2

Acredita-se que devem existir aproximadamente seis milhões de brasileiros com sinais e sintomas de DTM,2 sendo que a prevalência entre o sexo feminino é o dobro em relação ao sexo masculino.3 Esse achado é atribuído a fatores como constituição física, níveis hormonais 
e percepção diferente dos sintomas.3 Mulheres também freqüentam mais os consultórios odontológicos e este fator também pode influenciar este dado.4

As DTM's são resultados de alterações ocorridas tanto nos músculos mastigatórios quanto na própria articulação.1 No entanto, é possível destacar outros fatores mais amplos e complexos como a postura corporal e a mecânica ventilatória, que contribuem para ocorrência dessas disfunções.5 Estudos recentes têm verificado que a mecânica e o padrão ventilatório são capazes de exercer influência nos casos de DTMs.6 Achados sugerem uma possível relação entre a incidência de patologias respiratórias e DTM, referindo a obstrução como motivo de alterações na postura craniocervical e mandibular para facilitar a respiração e vencer a maior resistência das vias aéreas.5 Essa relação é especialmente determinada pelo uso excessivo dos músculos inspiratórios acessórios, decorrente basicamente de fatores como padrão ventilatório apical, tensão/ansiedade e respiração bucal.6

o distúrbio da mecânica respiratória pode levar ao uso inadequado dos músculos acessórios da respiração, resultando no desenvolvimento de alterações importantes da coluna cervical que, por sua vez, podem contribuir para o desencadeamento de alterações do sistema estomatognático.7 0 músculo esternocleidomastóideo (ECOM) é um dos principais responsáveis pelas alterações ocorridas na ATM. Este fato pode ser justificado por ser este um músculo atuante na flexão da cervical sobre a torácica que pode estar associada à anteriorização da cabeça. Esta anteriorização, por sua vez, pode tensionar a musculatura hióidea, tracionando a mandíbula posteriormente. Além disso, pode influenciar o esforço inspiratório, ocasionando uma maior utilização da musculatura acessória, o que aumenta a probabilidade de alterações no aparelho estomatognático.8

O Objetivo geral deste estudo é verificar a ocorrência de sinais e sintomas de disfunção temporomandibular em pacientes portadores de Doença Pulmonar Obstrutiva Crônica.

\section{Metodologia}

Estudo observacional de corte transversal realizado em indivíduos de ambos os sexos, com idade entre 40 e 65 anos, portadores de Doença Pulmonar Obstrutiva Crônica. Os participantes tinham diagnóstico da doença há pelo menos um ano, confirmado por profissional de medicina especializado em pneumologia e com laudo espirométrico (máximo 15 meses anteriores a data de coleta). Os indivíduos foram selecionados em um Ambulatório de Pneumologia de um Hospital Público da cidade de Salvador, Bahia.

Foram excluídos pacientes com doenças musculoesqueléticas sistêmicas que pudessem levar ao comprometimento das ATM's, com história de fraturas mandibulares ou cirurgia ortognática e os indivíduos que estivessem realizando tratamento ortodôntico. Também foram excluídos portadores de desordens neuromusculoesqueléticas crônicas que pudessem afetar a postura e o controle motor.

De acordo com o cálculo amostral, responsável por determinar o número necessário de pacientes para responder à pergunta de investigação, 30 pacientes voluntários portadores de DPOC foram selecionados, considerando-se para pesquisas exploratórias em nível laboratorial um alfa de $5 \%$ e precisão de $2 \%$ e atribuindo-se uma prevalência de $50 \%$. Essa seleção foi realizada através de uma abordagem verbal a partir do comparecimento às consultas previamente marcadas do Ambulatório de Pneumologia do referido hospital. Após os participantes tomarem ciência dos objetivos e procedimentos da pesquisa e assinarem o termo de consentimento livre e esclarecido, a coleta de dados foi iniciada.

Um questionário pré-testado foi aplicado para obter dados básicos sócio-demográficos (retirados do banco de itens do Instituto Brasileiro de Geografia e Estatística - IBGE). Dados clínicos relativos aos possíveis sinais e sintomas de DTM entre os indivíduos do estudo foram obtidos através do Questionário de Fonseca (1992).9,10 Foram recolhidos, ainda, dados nos prontuários referente aos resultados e data dos laudos espirométricos dos pacientes, elaborados previamente pela equipe de profissionais de um Ambulatório de referência da cidade de Salvador, Bahia, a fim de se obter o grau de comprometimento do indivíduo frente à 
Doença Pulmonar Obstrutiva Crônica. O estadiamento da doença foi estabelecido como DPOC Leve, Moderada e Severa.

A Cirtometria Torácica foi aplicada para identificar a mobilidade toraco-abdominal do indivíduo. Esse teste foi realizado com auxílio de uma fita métrica inextensível, com escala de 0 a 150 centímetros e precisão de 0,1 centímetro, apoiada junto à pele em três níveis perimétricos (na linha axilar, xifoidal e basal). Cada medida foi obtida após solicitar ao paciente que realizasse uma expiração forçada (até o volume residual), seguida de uma inspiração máxima e novamente uma expiração máxima. 0 coeficiente respiratório consistiu no valor entre as medidas obtidas com a fita métrica entre a inspiração e expiração máximas. As medidas foram realizadas e registradas duas vezes e considerada a média dos dois valores mensurados.

Como variável independente do estudo tem-se o grau de DPOC e como variável dependente, o grau de disfunção temporomandibular. A mobilidade torácica apresenta-se como co-variável do estudo.

Em caracterização por natureza, o sexo, estado civil, raça/cor, o tabagismo e estilismo apresentam-se com variáveis qualitativas nominais. 0 nível de escolaridade é considerado como qualitativa ordinal e o grau de DPOC e de DTM como qualitativas categóricas. As variáveis quantitativas discretas são: idade, que foi considerada em valores absolutos e quantidade de cigarros/dia, considerada em valores relativos.

A análise dos resultados foi feita através de estatística descritiva com uso de tabelas e gráficos contendo frequências e parâmetros de média e desvio padrão. Para verificar a associação entre as variáveis, foi utilizado o teste Exato de Fisher, nas análises univariadas considerando-se um alfa de 5\%. 0 pacote estatístico utilizado foi o Statistic Package for Social Science (SPSS) versão 14.0 para Windows.

Este trabalho seguiu as normas da resolução 196/96 e foi aprovado através do protocolo 137/2008, pelo Comitê de Ética em Pesquisa da Fundação Bahiana para Desenvolvimento das Ciências.

\section{Resultados}

Foram avaliados 30 indivíduos cuja mediana de idade equivale a 57 anos $(\mathrm{Q} 1=52 ; \mathrm{Q} 3=$ $60,5)$, havendo predominância do sexo feminino $(73,3 \%)$. Metade da população caracterizouse solteira e com nível de escolaridade baixo a médio (Tabela 1). A amostra foi constituída por pessoas que se definiram predominantemente como afrodescendentes $(66,7 \%)$, considerando as cores parda/mulata e preta.

As características clínicas da população estão expressas na Tabela 2. Apenas 10\% dos indivíduos não apresentaram sinais e sintomas de Disfunção Temporomandibular, sendo o grau moderado o de maior prevalência $(53,3 \%)$. Todos os participantes possuem história de tabagismo, tendo a maioria $(66,7 \%)$ se declarada como ex-fumante. A Figura 1 demonstra comparativamente a distribuição dos graus de DPOC e DTM.

Houve restrição de mobilidade toracoabdominal em todos os níveis cirtométricos avaliados, sendo apresentada por 27 indivíduos (86,67\%), no Nível Basal.

Aplicou-se o teste Exato de Fisher, para verificar associação entre o grau de Doença Pulmonar Obstrutiva Crônica (DPOC) e a mobilidade toracoabdominal, em seus níveis axiliar, xifoidal e basal. Conforme se pode observar na tabela 3, todos os participantes apresentaram algum grau de restrição de mobilidade tóracoabdominal, em algum dos níveis axilar, xifoidal ou basal. Considerando o nível axilar, dos indivíduos com DPOC Leve, 70\% possui mobilidade toracoabdominal normal enquanto apenas 23,1 e 28,6\% dos portadores de DPOC moderada e grave apresentaram esta normalidade. Nos níveis axilar e xifoidal, a restrição grave de mobilidade foi apresentada exclusivamente por portadores de DPOC da mesma categoria. No entanto, não houve significância estatística de associação entre estas variáveis.

Houve relação estatisticamente significativa entre os graus de DPOC e DTM, estando o grau moderado de DPOC em maior associação com o grau moderado de DTM $(92,6 \%)$. 


\section{Discussão}

Os resultados apresentados por este estudo indicam que o grau de sinais e sintomas de DTM apresentado está associado com a gravidade da DPOC, sugerindo uma possível relação entre a incidência de disfunção temporomandibular e a presença de patologias respiratórias, corroborando com os achados da literatura revisada. A literatura tem observado uma maior incidência de obstruções das vias aéreas em indivíduos com DTM comparado aos assintomáticos.5 Também tem sido observada uma maior atividade eletromiográfica dos músculos ECOM e trapézio durante a inspiração nasal de crianças respiradoras bucais do que em respiradoras nasais, conseqüência do maior esforço da musculatura acessória da respiração para vencer a resistência aumentada das vias aéreas superiores. 9 No entanto, a comparacão entre os resultados do presente estudo com os achados destes outros estudos deve ser feita com reserva, devido às diferenças fisiopatológicas entre as doenças obstrutivas avaliadas.

Foi utilizado, a fim de avaliar a presença e o grau de sinais e sintomas de disfunção temporomandibular, o Índice Anamnésico proposto por Fonseca.9,10 Este mostrou-se uma ferramenta simples e de boa aplicabilidade na população estudada. Trata-se de um instrumento adaptado para o Brasil, capaz de distinguir os graus de severidade das amostras estudadas.10 Além disso, sua simplicidade e auto-administração12 amplia as possibildades de uso à serviços públicos por qualquer membro da equipe de saúde, auxiliando nos levantamentos epidemiológicos e no controle de tratamento.

Todos os participantes do estudo apresentaram algum grau de restrição de mobilidade tóracoabdominal, seja esta nos níveis axilar, xifoidal ou basal. Foi observada, ainda, uma possível associação entre os graus de DPOC e de mobilidade tóracoabdominal. Um estudo em uma amostra de 30 portadores de DPOC com grau moderado e grave, observou a presença de restrição de mobilidade tóracoabdominal de quase $3 \mathrm{~cm}$, em média, na população. No entanto, este não fornece informações associativas entre o grau de DPOC e o de mobilidade.12

Embora ainda não seja possível uma explicação aprofundada sobre os resultados obtidos na análise da mobilidade torácica e da DPOC, é provável que a restrição desta mobilidade esteja relacionada à presença da hiperinsuflação pulmonar, o que leva o portador de DPOC a atingir praticamente seus limites elásticos de mecânica ventilatória.11,12 Comparações com populações hígidas podem auxiliar a compreender melhor esta questão.

A freqüência elevada de sintomas de DTM em 90\% dos pacientes estudados evidencia a similaridade entre os dados deste trabalho aos relatados em outros estudos que avaliaram alterações craniocervicais em portadores de doenças respiratórias obstrutivas. 7, 8, 11 e 13 Em 2005, por exemplo, um estudo demonstrou uma prevalência de 93,4 \% de DTM, sob avaliação através do Índice Anamnésico de Fonseca, em crianças asmáticas. 7 Esta alta prevalência pode ser resultado do uso inadequado dos músculos acessórios da respiração (frequente em portadores de distúrbios respiratórios), que acarreta o desenvolvimento de alterações importantes da coluna cervical e consequentemente ao sistema estomatognático.

O grau Moderado de Disfunção Temporomandibular foi o mais prevalente entre a população (53,3\%). Este achado é compatível com os resultados encontrados por pesquisadores que avaliaram a mecânica ventilatória de indivíduos com DTM e assintomáticos. 5 Estes relataram uma prevalência de $45 \%$ de sinais e sintomas de DTM Moderada, seguida por graus Leve e Severo. No entanto, achados controversos encontraram como mais prevalente o Grau Leve de sinais e sintomas de DTM (63\%).7

0 presente estudo apresenta como principais limitações a ausência de uma avaliação clínica e não somente anamnésica, para melhor caracterizar a presença de Disfunção Temporomandibular em portadores de DPOC. A análise, ainda, de um grupo controle (não portadores da doença) deve ser incrementada a estudos posteriores, a fim de identificar a susceptibilidade de portadores de DPOC ao desenvolvimento de DTM. 


\section{Conclusão}

Pode-se, portanto, concluir que existe uma alta prevalência de Disfunção Temporomandibular em indivíduos portadores de Doença Pulmonar Obstrutiva Crônica, sendo o grau moderado o mais prevalente.

O grau de sinais e sintomas de DTM está associado com a gravidade da DPOC. Embasados nos resultados deste estudo, os programas de fisioterapia voltados aos portadores de DPOC devem incrementar aos cuidados cardiorrespiratórios, a atenção aos sinais e sintomas de DTM.

\section{Referências}

1. OKESON, JP. Dor Orofacial - Guia de avaliação, diagnóstico e tratamento. São Paulo: Quintessense, 1998.

2. SHIBAYAMA, R.; GARCIA, AR.; ZUIM, PRJ. Prevalência de desordem temporomandibular (DTM) em pacientes portadores de próteses totais duplas, prótese parciais removíveis e universitários. Revista de Odontologia Araçatuba, v.25, n.2, p. 18-21, Jul/Dez 2004.

3. TEIXEIRA, MJ. Dor: Contexto Interdisciplinar. Curitiba: Ed. Maio, 2003.

4. SELAIMEN, C.; BRILHANTE, DP.; GROSSI, ML.; GROSSI, PK. Avaliação da depressão e de testes neuropsicológicos em pacientes com desordens temporomandibulares. Ciência \& Saúde Coletiva, v.12, n.6, 1629-1639, 2007.

5. PASINATO F.; CORRÊA ECR.; PERONI ABF. Avaliação da Mecânica Ventilatória em indivíduos com disfunção temporomandibular e assintomáticos. Revista Brasileira de Fisioterapia, São Carlos, v.10, n.3, 285-289, jul./set 2006.

6. CORRÊA, ECR.; BÉRZIN, F. Temporomandibular disorder and dysfunctional breathing. Brazil Journal Oral Science. v. 03, n. 10, jul/set 2004.

7. CHAVES, TC.; COSTA, D.; GROSSI, D.B.; BERTOLLI, F. Avaliação anamnésica de sintomas de disfunção temporomandibular em crianças asmáticas. Fisioterapia e Pesquisa vol.11, n 1, jan/abr 2005.

8. RIBEIRO, E.C. Estudo eletromiográfico dos músculos esternocleidomastóideo e trapézio em crianças respiradoras bucais e nasais. Dissertação de Mestrado. Santa Maria: Univ. Federal de Santa Maria; 2000.

9. FONSECA, DM. Disfunçäo craniomandibular - (DCM): diagnóstico pela anamnese [Tesis de Maestría]. Säo Paulo: Universidade de Säo Paulo, Faculdade de Odontologia de Bauru, 1992.

10.LAZARO, J.; ALVARADO, S. Validation of Fonseca's Simplified Index for Temporomandibular Disorder's Diagnosis. Odontologia. Clínica.-Científica, Recife, 8 (2), 163-168, abr/jun 2009.

11.GONZALEZ, DAB.; ANDRADE, DV.; GONZALEZ, TO. Correlação entre disfunção temporomandibular, postura e qualidade de Vida. Revista Brasileira de Desenvolvimento Humano, v.8, n.1, 79-86 2008.

12.PAULIN, E.; BRUNETTO, AF.; CARVALHO, CRF. Efeitos de programa de exercícios físicos direcionados ao aumento da mobilidade torácica em pacientes portadores de doença pulmonar obstrutiva crônica. Jornal de Pneumologia, v.29, n.5, set/out 2003.

13. SCANLAN WS. Fundamentos da Terapia Respiratória de EGAN. 7a Elselvier, 2000.

14. SILVA FB, SAMPAIO LMM, CARRASCOSA AC. Avaliação Fisioterapêutica dos sistemas mastigatório e respiratório de um portador de Sindrome Otodental: Um estudo de caso. Revista Brasileira de Fisioterapia, v.10, n.1, 133-136, 2006. 


\section{Anexos: Tabelas e Figuras}

Tabela 1 - Características sociodemográficas dos portadores de DPOC na cidade de Salvador, Ba.

\begin{tabular}{|c|c|c|}
\hline Variáveis & $\mathrm{N}=30$ & $\%$ \\
\hline \multicolumn{3}{|l|}{ Sexo } \\
\hline Masculino & 8 & 26,7 \\
\hline Feminino & 22 & 73,3 \\
\hline Idade (em anos) 1 & 56,33 & $(52-60,5)$ \\
\hline \multicolumn{3}{|l|}{ Raça/Cor } \\
\hline Branca & 3 & 10,0 \\
\hline Mulata & 12 & 40,0 \\
\hline Negra & 8 & 26,7 \\
\hline Amarela (oriental ou indígena) & 7 & 23,3 \\
\hline \multicolumn{3}{|l|}{ Estado civil } \\
\hline Solteiro(a) & 15 & 50,0 \\
\hline Casado(a) & 10 & 33,3 \\
\hline Separado(a) / divorciado(a) & 3 & 10,0 \\
\hline Viúvo(a) & 2 & 6,7 \\
\hline \multicolumn{3}{|l|}{ Escolaridade } \\
\hline Analfabeto & 1 & 3,3 \\
\hline Lê e escreve, mas nunca freqüentou escola & 3 & 10,0 \\
\hline $1^{\circ}$ grau completo & 15 & 50,0 \\
\hline $2^{\circ}$ grau completo & 10 & 33,4 \\
\hline Nível superior & 1 & 3,3 \\
\hline
\end{tabular}

Foi estimada a mediana e os quartis (Q1/4 - Q3/4).

Tabela 2 - Características clínicas dos portadores de DPOC na cidade de Salvador, Ba.

\begin{tabular}{lrr}
\hline Variáveis & $\mathrm{N}=30$ & $\%$ \\
\hline Grau da Doença Pulmonar Obstrutiva Crônica (DPOC) & 33,3 \\
$\quad$ Leve & 10 & 43,4 \\
Moderado & 13 & 23,3 \\
Grave & 7 & \\
& & \\
Grau da Disfunção Temporomandibular (DTM) * & 18,5 \\
Leve & 5 & 53,3 \\
Moderado & 16 & 20 \\
Grave & 6 & \\
Tabagismo & & 0 \\
Não & 0 & 33,3 \\
Sim & 10 & 66,7 \\
Ex-fumante & 20 &
\end{tabular}

Cigarros por dia $* * \quad 20(17,5-20)$

* Três pacientes $(10,0 \%)$ não apresentaram DTM.

** Foi estimada a mediana e os quartis (Q1/4 - Q3/4). 
Tabela 3 - Relação entre o grau de Doença Pulmonar Obstrutiva Crônica (DPOC) e a mobilidade toracoabdominal dos portadores de DPOC, na cidade de Salvador, BA

\begin{tabular}{|c|c|c|c|c|c|c|}
\hline \multirow[t]{3}{*}{ Mobilidade Toracoabdominal } & \multicolumn{6}{|c|}{ (DPOC) } \\
\hline & & Leve & \multicolumn{2}{|c|}{ Moderada } & \multicolumn{2}{|c|}{ Grave } \\
\hline & $\mathrm{n}=10$ & $\%$ & $\mathrm{n}=13$ & $\%$ & $\mathrm{n}=7$ & $\%$ \\
\hline \multicolumn{7}{|l|}{ Nível Axilar $(\mathrm{p}=0,100)$} \\
\hline Restrição Leve & 3 & 30 & 9 & 69,2 & 4 & 57,1 \\
\hline Restrição Moderada & 0 & 0 & 1 & 7,7 & 0 & 0 \\
\hline Restrição Grave & 0 & 0 & 0 & 0 & 1 & 14,3 \\
\hline Sem Restrição & 7 & 70 & 3 & 23,1 & 2 & 28,6 \\
\hline \multicolumn{7}{|l|}{ Nível Xifoidal ( $p=0,227)$} \\
\hline Restrição Leve & 4 & 40 & 7 & 53,8 & 3 & 42,9 \\
\hline Restrição Moderada & 1 & 10 & 4 & 30,8 & 0 & 0 \\
\hline Restrição Grave & 0 & 0 & 0 & 0 & 1 & 14,3 \\
\hline Sem Restrição & 5 & 50 & 2 & 15,4 & 3 & 42,9 \\
\hline \multicolumn{7}{|l|}{ Nível Basal ( $p=0,203)$} \\
\hline Restrição Leve & 3 & 30 & 4 & 30,8 & 2 & 28,6 \\
\hline Restrição Moderada & 2 & 20 & 8 & 61,5 & 3 & 42,9 \\
\hline Restrição Grave & 2 & 20 & 1 & 7,7 & 2 & 28,6 \\
\hline Sem Restrição & 3 & 30 & 0 & 0 & 0 & 0 \\
\hline
\end{tabular}

P-valor obtido através do teste Exato de Fisher.

Tabela 4 - Relação entre o grau de Doença Pulmonar Obstrutiva Crônica (DPOC) e o Grau de disfunção Temporomandibular dos portadores de DPOC, na cidade de Salvador, BA.

\begin{tabular}{lrrrrrr}
\hline DTM & \multicolumn{2}{c}{$\begin{array}{c}\text { DPOC } \\
\text { LeveModerada } \\
(\mathrm{n}=10)\end{array}$} & $\begin{array}{c}\text { Grave } \\
(\mathrm{n}=13)\end{array}$ & $\%$ & $(\mathrm{n}=7)$ & $\%$ \\
\hline Leve & 4 & $40^{*}$ & 0 & 0 & 1 & 14,3 \\
Moderada & 2 & 20 & 12 & $92,3^{*}$ & 2 & 28,6 \\
Grave & 1 & 10 & 1 & 7,7 & 4 & $57,1^{*}$ \\
\hline
\end{tabular}

$\mathrm{n}$ = Número de observações; P-valor obtido através do teste Exato de Fisher. * p < 0, 05
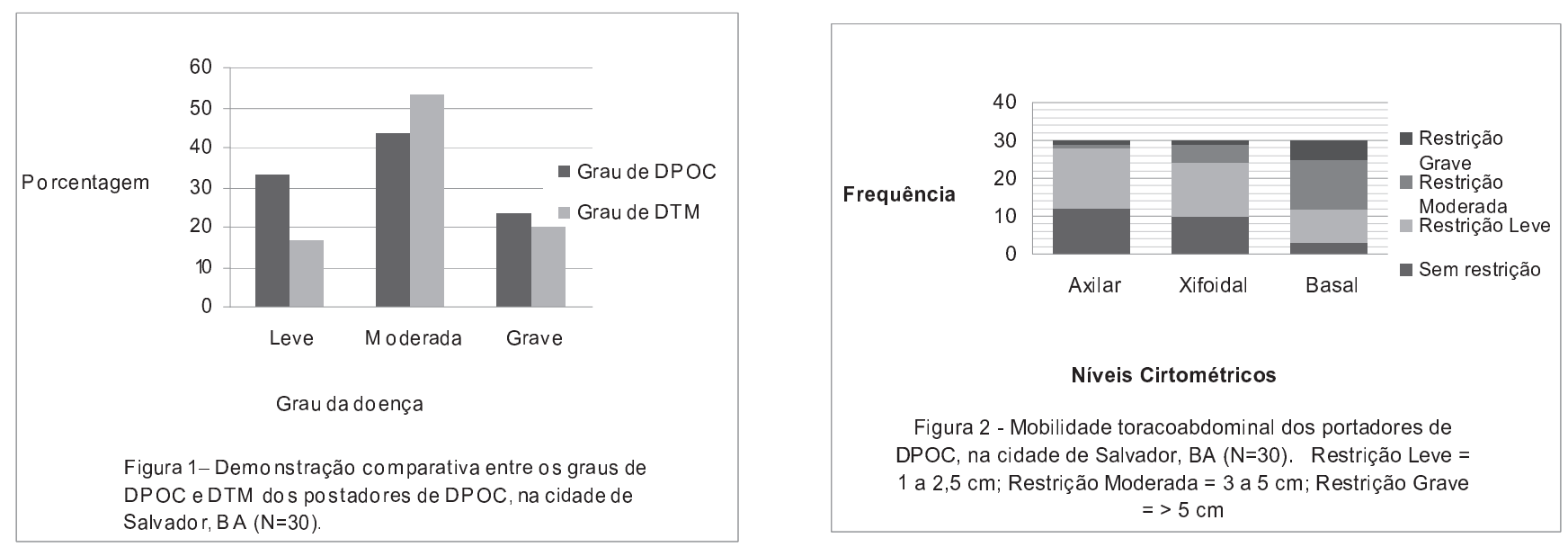